\title{
The Effects of Hippotherapy on Standing Balance in Patients with Incomplete Cervical Spinal Cord Injuries: A Pilot Study
}

\author{
Bo-Ra Choi ${ }^{1}$, Ju-Hyun Kim ${ }^{1}$, Jeong-Uk Lee ${ }^{1}$, Junghwan Kim ${ }^{2 *}$ \\ ${ }^{1}$ Laboratory of Health Science \& Nanophysiotherapy, Department of Physical Therapy, Graduate School, Yongin University, Yongin, \\ Korea; ${ }^{2}$ Department of Physical Therapy, College of Public Health \& Welfare, Yongin University, Yongin, Korea. \\ Email: junghwankim3@yongin.ac.kr
}

Received January $4^{\text {th }}, 2013$; revised February $6^{\text {th }}, 2013$; accepted February $2^{\text {th }}, 2013$

\begin{abstract}
The present study was designed to evaluate the effects of hippotherapy on standing balance in patients with incomplete cervical spinal cord injuries. Ten patients (8 men and 2 women) who were diagnosed with cervical levels of injury between 3 and 6, and D according to the ASIA Impairment Scale. All patients were tested by the physiotherapists prior to and after hippotherapy treatment. Standing balance was measured using the Interactive Balance System (IBS). The weight distribution index was significantly increased in application of hippotherapy compared with control groups in patients with a neurologic level of cervical 3 and 4, and with Brown-Sequard syndrome, respectively. However, most of the patients exhibited no significant changes in the standing balance index, such as F1, F2-4, F5-6, F7-8, and SI scores, after hippotherapy. These results suggest that hippotherapy did not have a significant effect on the standing balance of patients with incomplete cervical spinal cord injuries.
\end{abstract}

Keywords: Spinal Cord Injury; Standing Balance; Hippotherapy

\section{Introduction}

Direct or indirect damage to the central or peripheral nervous system can reduce an individual's ability to perform activities of daily living [1-3]. In a significant number of spinal cord injured individuals, incomplete injury to the spinal cord may spare their descending motor control pathways and allow them to retain the ability to walk [4]. However, one of the key functional deficits is poor balance; people who have sustained a spinal cord injury (SCI) lack the normal postural synergies and sensory-motor integration of the lower limbs and trunk that regulate upright position [5]. Balance is a somewhat ambiguous term used to describe the ability to maintain or move within a weight-bearing posture without falling [6]. Balance is a significant issue for the majority of ambulatory patients with incomplete spinal cord injuries. Indeed, they are at increased risk of falling [7]. Because of these individuals' poor balance, bone fractures are common [8]. The mortality rate associated with falls increases dramatically with injury. In addition, instability and falls can be markers of poor health and declining function, and they are often associated with significant morbidity [6]. One potential way to improve balance and mobility and

*Corresponding author. to reduce falls is through exercise interventions [9]. The muscle groups of the lumbar-pelvis-hip complex (an aspect of the neuromuscular system) can be targeted in an exercise intervention program to improve static postural stability and assist patients in returning to equilibrium after perturbation [10]. According to Nadler et al., the focus of such a training regimen should also include muscular stabilization of the abdominal, paraspinal, and gluteal muscles to provide better stability and control [11]. However, central neuropathies such as spinal cord injuries and strokes usually reduce the strength of these core muscles and their corresponding functional ability required for standing [12]. To our knowledge, strengthening the lumbar-pelvis-hip muscles should improve balance and stability and thus reduce one's likelihood of falling. Hippotherapy is a physical treatment strategy in which the movement of a horse is used to improve the posture, balance, and general development of people with or without motor difficulties [13]. In addition, rhythmic equine movement is used in hippotherapy to treat people with neurologic impairments. When a horse walks, its movement involves a delicate coordination of neck, trunk, and limb muscles. Several studies have reported hippotherapy's positive impact on muscle tone, posture, 
balance as well as its psychosomatic influence on patients [14-16]. The three-dimensional movement of a horse's back as it walks stimulates a three-dimensional movement in the rider's pelvis that is likened to the movement of the human pelvis that occurs during walking. Thus, because of this similarity in pelvic movement during riding to that which occurs when ambulating, several authors have demonstrated improvements in patients' gait post hippotherapy. According to Bertoti [17], horseback riding can improve the spine, trunk, and pelvis, especially in children with spasticity diplegia. For several years, hippotherapy has been used in the rehabilitation of spinal cord injured patients, and positive effects on spasticity and well being have been reported $[14,18]$. Unfortunately, only a few studies assessing the effect of hippotherapy on spasticity in spinal cord injured patients have been conducted, and not apply to the existing literature of standing balance in spinal cord injured patients. Studies that aim to explain the mechanisms underlying the effects of hippotherapy on standing balance are even scarcer. Therefore, the aim of this study was to investigate hippotherapy's effect on the standing balance of incomplete cervical spinal cord injury patients. We assessed 10 incomplete cervical spinal cord injured patients in our study, and the physiotherapist in charge rated the standing balance of the patients prior to and immediately following hippotherapy, according to the interactive balance system.

\section{Materials and Methods}

Ten patients (8 men and 2 women) were recruited from $\mathrm{S}$ rehabilitation center in Gyeonggi-do, Korea. They had spinal cord injuries that had occurred less than one year prior to the time of the study. Participants had been previously diagnosed with incomplete cervical spinal cord injuries according to established criteria. They received written and verbal explanations about the purposes and procedures of the study. Inclusion criteria were as follows: 1) able to standing independently without an assistive device for 32 seconds; 2) able to standing independently with both eyes closed for 32 seconds; 3) no skin problems or wounds; and 4) no horseback riding for six months leading up to the study. Exclusion criteria were as follows: 1) pain during neck active motion; and 2) dizziness with both eyes closed in a standing posture. All patients were tested by the physiotherapists prior to and after hippotherapy treatment. They ranged in age between 27 and 53 years (mean $38.4 \pm 10.6$ ). The neurologic levels of their cervical cord injuries were between 3 and 6, and D according to the ASIA Impairment Scale [19]. In clinical practice, each hippotherapy session lasted for 25 - 30 minutes [14]. The treatments were performed using hippotherapy. We set the treatment speed at
65 and the mode at 58 to improve the lumbar-pelvis-hip muscles (Figure 1). The physiotherapist involved in the hippotherapy intervention helped with transfers and remained with the subjects throughout each session. Participants were evaluated in each session. Each session included the assessment of static standing balance using the Interactive Balance System (IBS) (Tetrax Inc., Israel) (Figure 1). This system uses four separate platforms, each measuring the vertical pressure fluctuations induced by the two heels and toe parts, respectively [20-22]. A pattern analysis of the postural performance of the above measurements was conducted at the following eight positions: head straight with eyes open (NO); head straight with eyes closed (NC); standing on elastic pads with eyes open (PO); standing on elastic pads with eyes closed (PC); head right with eyes closed (HR); head left with eyes closed (HL); head up with eyes closed (HB); and head down with eyes closed (HF). Fourier transformations were derived from four independent wave signals and presented in the form of a spectrum. These frequency bands were divided into four frequency signals: low (0.01 - $0.1 \mathrm{~Hz})$, medium-low (0.1 - $0.5 \mathrm{~Hz})$, medium-high $(0.5-1.0 \mathrm{~Hz}$ ), and high (1.0 Hz and above). F1 (low frequency; $0.01-0.1 \mathrm{~Hz}$ ) frequencies are linked visual control, and they typically dominate normal steady and
(A) (a) IBS

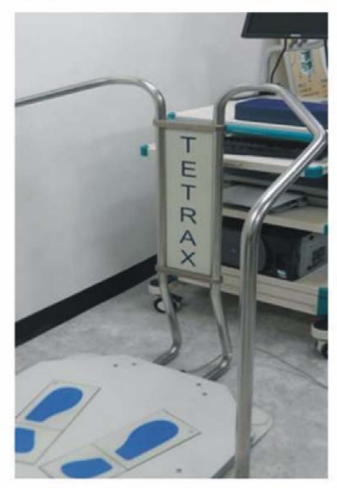

(b) CCS

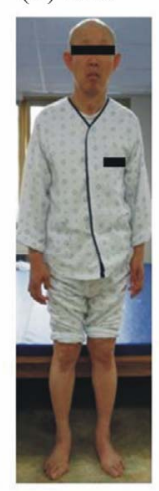

(c) BSS

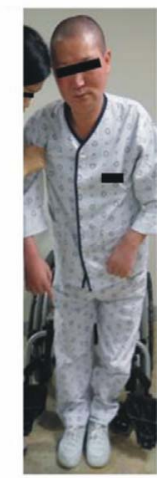

(B) Hippotherapy

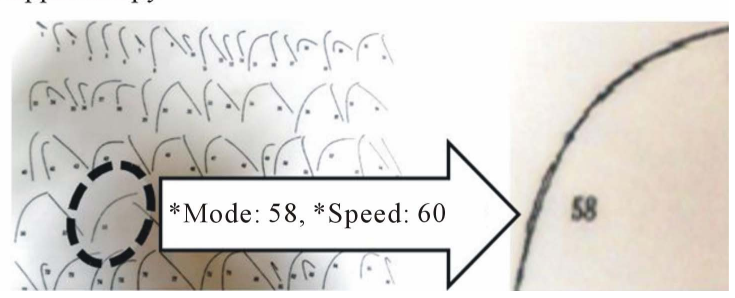

Figure 1. Measurements of standing balance and application of hippotherapy in patients with incomplete cervical spinal cord injury. The explanation of the interactive balance system (A-a) and the application of hippotherapy using the mode 58 and speed 65 (B), as described in the Materials and Methods. IBS, interactive balance system; CCS, patients with central cord syndrome; BSS, patients with Brown-Sequard syndrome. 
undisturbed posture. F2-4 (medium-low; $0.1-0.5 \mathrm{~Hz}$ ) frequencies are sensitive to vestibular stress and disturbances. F5-6 (medium-high; $0.5-1.0 \mathrm{~Hz}$ ) frequencies reflect the somatosensory activity of the lower extremities and spine. F7-8 (high; $1.0 \mathrm{~Hz}$ and above) frequencies are often induced by dysfunctions in the central nervous system. The weight distribution index (WDI) was measured of weight distribution over the four platforms. The stability index (SI) represents the status of an individual's general postural stability [20]. All patients provided their informed consent to participation in the study, and the protocol for the study was approved by the Committee of Ethics in Research of the University of Yongin, in agreement with the terms of Resolution 5-1-20, December 2006. Standing balance was evaluated twice, pre- and post-intervention. The existence of a statistical difference between standing balance before and after hippotherapy treatment was assessed by performing Wilcoxon's signedrank test. Statistical significance was set at the 5\% level. SPSS for Windows (version 12.0) was used for analysis in this study.

\section{Results}

Table 1 presents details about the participants. The weight distribution index (WDI) was significantly increased in application of hippotherapy compared with control groups in patients with a neurologic level of cervical 3 and 4 (before hippotherapy: 7.6 \pm 2.9, after hippotherapy: $8.9 \pm$ 2.9) (Figure 2), and with Brown-Sequard syndrome (before hippotherapy: $7.3 \pm 3.3$, after hippotherapy: $9.2 \pm$ 3.3) (Figure 3), respectively. However, most of the patients exhibited no significant changes in the standing balance index, such as F1, F2-4, F5-6, F7-8, and stability index (SI) scores, after hippotherapy (Figures 2 and 3 and Tables 2-4).

\section{Discussion}

Various case studies have demonstrated support for the theory that therapeutic hippotherapy can be helpful for patients with neurologic impairments. A previous study with nine multiple sclerosis patients reported that hippotherapy resulted in statistically significant improvements in patients' Berg Balance Scale (BBS) and Performance-Oriented Mobility Assessment (POMA) scores [15]. Hammer et al. also reported significant improvements in three out of 11 patients using the BBS and, when a qualitative measure was included in the analysis, balance improvements were reported in eight patients [23]. The present study is the first objective clinical analysis of the effects of hippotherapy on standing balance in patients with incomplete cervical spinal cord injuries. In contrast to other studies, we used the computerized Interactive Balance System and found non-sig-

Table 1. General characteristics of patients with incomplete cervical spinal cord injury.

\begin{tabular}{|c|c|}
\hline Variable & SCI patients with hippotherapy \\
\hline Age & $38.4 \pm 10.6$ years \\
\hline \multicolumn{2}{|l|}{ Sex } \\
\hline Female (\%) & $2(20)$ \\
\hline Male (\%) & $8(80)$ \\
\hline Height & $171.6 \pm 7.1 \mathrm{~cm}$ \\
\hline Weight & $65.8 \pm 8.6 \mathrm{~kg}$ \\
\hline BMI & $21.9 \pm 1.1 \mathrm{~kg} / \mathrm{m}^{2}$ \\
\hline \multicolumn{2}{|l|}{ Past history } \\
\hline Hypertension & - \\
\hline Diabetes mellitus & - \\
\hline
\end{tabular}

Times since SCI

Mechanism of injury

Fall (\%)

Assault (\%)

Injury level

Cervical 3/4 (\%)

Cervical 5/6 (\%)

SCI clinical syndrome

Central cord syndrome (\%)

Brown-Sequard syndrome (\%)

Surgery lesion

Anterior (\%)

Posterior

Mean \pm Standard Error, SCI, spinal cord injury; BMI, body mass index.

nificant into eight ranges of frequencies increasing postural sway. The null hypothesis was that patients with incomplete cervical spinal cord injuries would have significantly improved standing balance after hippotherapy treatment. However, according to our results, patients' standing balance index scores after hippotherapy intervention were not significantly improved. Manella and Backus [24] reported that gait deviations commonly observed after SCI include inadequate hip extension when standing as a result of tightness or spasticity of the iliopsoas muscle. The primary causes of pelvic anterior tilt include hip extensor, abdominal weakness, and hip flexor spasticity. The forward and backward trunk movements induced by hippotherapy may be affecting pelvic and hip 
(A) Total

(a)

(b)
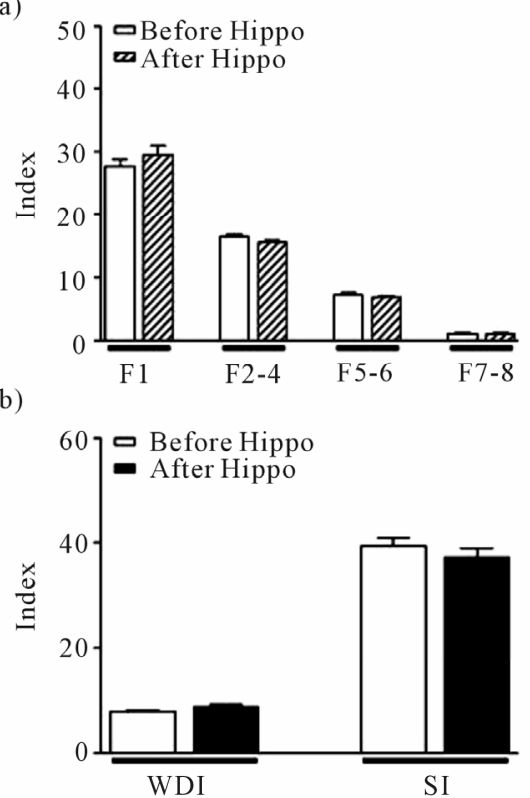

(B) C3/C4 injury

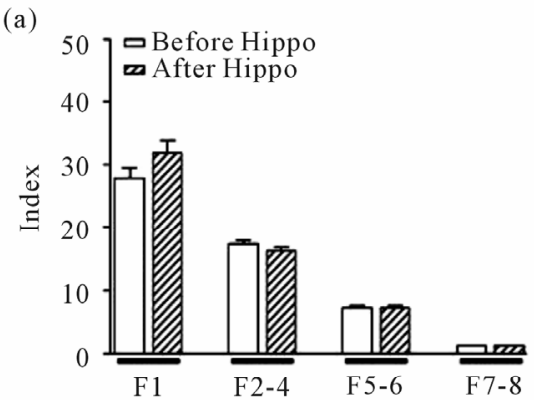

(b)

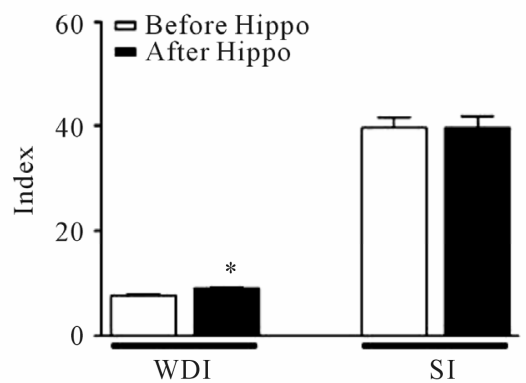

(C) C5/C6 injury

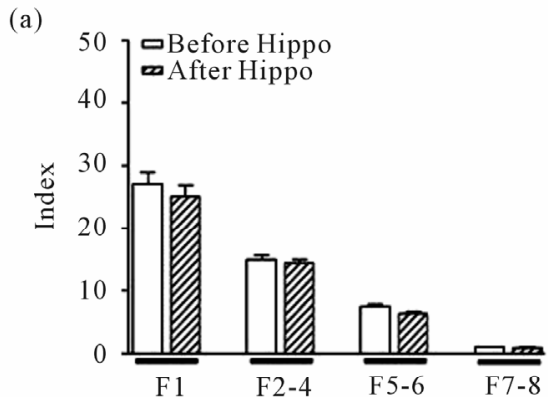

(b)

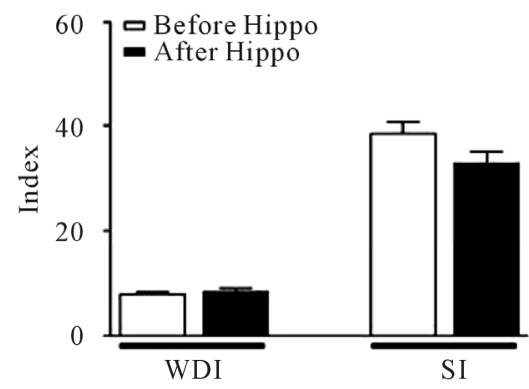

Figure 2. Effects of hippotherapy in patients with incomplete cervical spinal cord injury. (A) Standing balance indexes such as F1, F2-4, F5-6, F7-8, WDI, and SI were analyzed in sum total of cervical 3/4 and cervical 5/6 levels before and after hippotherapy (Total). Standing balance indexes were analyzed in levels of cervical 3/4 (B)(C3/C4 injury) and 5/6 (C)(C5/C6 injury) with or without hippotherapy, respectively. The F1 are linked visual control system. The F2-4 are sensitive to vestibular stress and disturbances. The F5-6 reflect somatosensory activity by the lower extremities and spine. The F7-8 are linked central nervous system. The weight distribution index (WDI) was measured for weight distribution. The stability index (SI) represents the status of the general postural stability. "Significant differences between before and after pelvic exercise with $p$ $<$ 0.05. Hippo, hippotherapy.
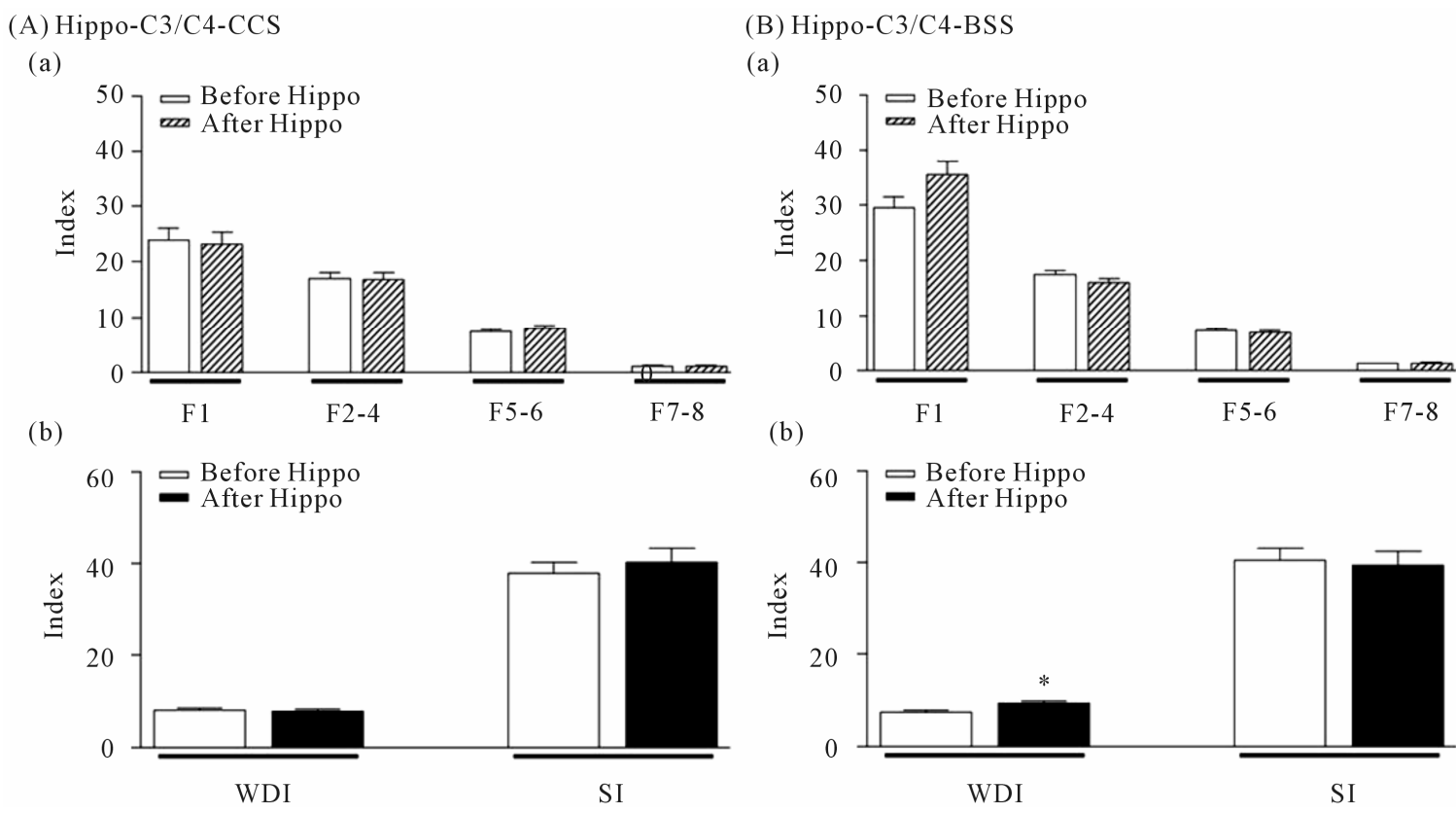

Figure 3. Effects of hippotherapy in levels of cervical $3 / 4$ in patients with central cord syndrome and Brown-Sequard syndrome. Standing balance indexes were analyzed in levels of cervical 3/4 with central cord syndrome (A, Hippo-C3/C4-CCS) and Brown-Sequard syndrome (B, Hippo-C3/C4-BSS), respectively. "Significant differences between before and after pelvic exercise with $\mathbf{p}<0.05$. Hippo, hippotherapy; CCS, patients with central cord syndrome; BSS, patients with Brown-Sequard syndrome. 
Table 2. The index of standing balance (F1, F2-4) before and after hippotherapy in patients with incomplete cervical spinal cord injury.

\begin{tabular}{|c|c|c|c|c|c|c|c|c|c|c|}
\hline & \multicolumn{10}{|c|}{$\mathrm{F} 1(0.35-0.50) \mathrm{Hz}$} \\
\hline & \multicolumn{2}{|c|}{ Total } & \multicolumn{2}{|c|}{ C3-4 } & \multicolumn{2}{|c|}{ C3-4, CCS } & \multicolumn{2}{|c|}{ C3-4, BSS } & \multicolumn{2}{|c|}{ C5-6 } \\
\hline & \multicolumn{2}{|c|}{ Hippotherapy } & \multicolumn{2}{|c|}{ Hippotherapy } & \multicolumn{2}{|c|}{ Hippotherapy } & \multicolumn{2}{|c|}{ Hippotherapy } & \multicolumn{2}{|c|}{ Hippotherapy } \\
\hline & Before & After & Before & After & Before & After & Before & After & Before & After \\
\hline NO & $19.4 \pm 7.9$ & $19.4 \pm 13.9$ & $21.8 \pm 9.0$ & $21.5 \pm 16.6$ & $16.8 \pm 4.1$ & $15.4 \pm 7.6$ & $23.9 \pm 10.0$ & $24.0 \pm 19.3$ & $15.2 \pm 2.7$ & $15.4 \pm 8.0$ \\
\hline NC & $24.8 \pm 7.6$ & $27.7 \pm 15.0$ & $25.9 \pm 8.0$ & $31.4 \pm 17.9$ & $23.7 \pm 0.1$ & $22.2 \pm 3.1$ & $26.9 \pm 9.6$ & $35.1 \pm 20.4$ & $22.9 \pm 7.5$ & $21.2 \pm 5.1$ \\
\hline $\mathrm{PO}$ & $22.8 \pm 8.6$ & $23.2 \pm 10.0$ & $22.6 \pm 9.8$ & $24.0 \pm 12.2$ & $20.0 \pm 6.7$ & $13.0 \pm 6.3$ & $23.7 \pm 11.3$ & $28.4 \pm 11.4$ & $23.3 \pm 7.4$ & $21.9 \pm 5.2$ \\
\hline PC & $37.0 \pm 17.5$ & $42.4 \pm 11.9$ & $34.6 \pm 20.9$ & $42.7 \pm 13.5$ & $27.5 \pm 15.4$ & $27.3 \pm 9.0$ & $37.4 \pm 23.7$ & $48.9 \pm 9.2$ & $41.2 \pm 10.3$ & $41.9 \pm 10.4$ \\
\hline HR & $28.5 \pm 6.6$ & $33.2 \pm 15.1$ & $30.0 \pm 7.6$ & $37.6 \pm 17.1$ & $27.9 \pm 5.2$ & $28.5 \pm 7.3$ & $30.8 \pm 8.7$ & $41.3 \pm 19.1$ & $25.8 \pm 4.3$ & $25.5 \pm 6.8$ \\
\hline HL & $30.9 \pm 6.6$ & $27.8 \pm 15.2$ & $32.1 \pm 7.8$ & $31.6 \pm 17.1$ & $31.3 \pm 4.0$ & $26.4 \pm 4.1$ & $32.4 \pm 9.3$ & $33.7 \pm 20.3$ & $29.0 \pm 4.0$ & $21.2 \pm 9.5$ \\
\hline HB & $26.1 \pm 9.9$ & $28.0 \pm 10.1$ & $24.2 \pm 8.8$ & $30.0 \pm 12.0$ & $22.1 \pm 16.7$ & $28.1 \pm 12.4$ & $25.1 \pm 6.6$ & $30.8 \pm 13.2$ & $30.0 \pm 12.1$ & $24.3 \pm 5.0$ \\
\hline \multirow[t]{5}{*}{$\mathrm{HF}$} & $31.3 \pm 12.7$ & $33.6 \pm 11.2$ & $31.9 \pm 14.1$ & $36.0 \pm 10.6$ & $22.3 \pm 10.8$ & $24.8 \pm 11.1$ & $35.8 \pm 14.3$ & $40.5 \pm 7.0$ & $30.2 \pm 11.9$ & $29.4 \pm 12.4$ \\
\hline & \multicolumn{10}{|c|}{$\mathrm{F} 2-4(0.50-0.75) \mathrm{Hz}$} \\
\hline & \multicolumn{2}{|c|}{ Total } & \multicolumn{2}{|c|}{ C3-4 } & \multicolumn{2}{|c|}{ C3-4, CCS } & \multicolumn{2}{|c|}{ C3-4, BSS } & \multicolumn{2}{|c|}{ C5-6 } \\
\hline & \multicolumn{2}{|c|}{ Hippotherapy } & \multicolumn{2}{|c|}{ Hippotherapy } & \multicolumn{2}{|c|}{ Hippotherapy } & \multicolumn{2}{|c|}{ Hippotherapy } & \multicolumn{2}{|c|}{ Hippotherapy } \\
\hline & Before & After & Before & After & Before & After & Before & After & Before & After \\
\hline NO & $11.0 \pm 5.6$ & $11.0 \pm 4.3$ & $10.9 \pm 5.9$ & $11.5 \pm 4.4$ & $9.8 \pm 4.0$ & $10.7 \pm 3.1$ & $11.4 \pm 6.6$ & $11.9 \pm 4.9$ & $11.0 \pm 5.2$ & $10.2 \pm 4.2$ \\
\hline NC & $16.3 \pm 5.1$ & $16.6 \pm 7.1$ & $17.5 \pm 5.3$ & $17.4 \pm 8.4$ & $18.9 \pm 5.7$ & $19.3 \pm 7.2$ & $16.9 \pm 5.2$ & $16.7 \pm 9.0$ & $14.2 \pm 4.2$ & $15.2 \pm 3.9$ \\
\hline $\mathrm{PO}$ & $12.7 \pm 5.4$ & $12.0 \pm 5.3$ & $12.5 \pm 5.0$ & $12.3 \pm 5.4$ & $13.8 \pm 5.8$ & $9.8 \pm 4.1$ & $11.9 \pm 4.8$ & $13.4 \pm 5.6$ & $13.2 \pm 6.2$ & $11.4 \pm 5.3$ \\
\hline PC & $21.8 \pm 6.2$ & $22.2 \pm 8.1$ & $22.5 \pm 6.6$ & $23.3 \pm 8.9$ & $21.3 \pm 6.0$ & $23.8 \pm 8.9$ & $23.0 \pm 7.0$ & $23.2 \pm 9.3$ & $20.5 \pm 5.3$ & $20.4 \pm 6.2$ \\
\hline HR & $17.8 \pm 6.3$ & $15.4 \pm 4.6$ & $19.4 \pm 6.4$ & $16.0 \pm 5.2$ & $18.9 \pm 6.1$ & $17.0 \pm 6.4$ & $19.5 \pm 6.7$ & $15.6 \pm 4.6$ & $15.2 \pm 5.5$ & $14.4 \pm 3.5$ \\
\hline HL & $16.8 \pm 6.4$ & $15.4 \pm 6.3$ & $18.4 \pm 5.6$ & $16.1 \pm 7.1$ & $17.3 \pm 4.9$ & $19.9 \pm 9.6$ & $18.9 \pm 5.9$ & $14.6 \pm 5.5$ & $14.0 \pm 7.0$ & $14.2 \pm 4.6$ \\
\hline HB & $18.9 \pm 9.2$ & $17.1 \pm 8.4$ & $20.2 \pm 10.2$ & $18.1 \pm 9.4$ & $18.5 \pm 8.5$ & $18.6 \pm 14.2$ & $20.8 \pm 11.1$ & $17.9 \pm 7.3$ & $16.8 \pm 7.0$ & $15.3 \pm 6.5$ \\
\hline $\mathrm{HF}$ & $16.3 \pm 5.7$ & $14.6 \pm 5.1$ & $17.4 \pm 6.3$ & $15.1 \pm 5.6$ & $18.1 \pm 5.9$ & $15.9 \pm 6.3$ & $17.1 \pm 6.7$ & $14.8 \pm 5.6$ & $14.5 \pm 4.2$ & $13.6 \pm 4.2$ \\
\hline
\end{tabular}

Abbreviations: CCS, central cord syndrome; BSS, Brown-Sequard syndrome; NO, head straight with eyes open; NC, head straight with eyes closed; PO, standing on elastic pads with eyes open; PC, standing on elastic pads with eyes closed; HR, head right with eyes closed; HL, head left with eyes closed; HB, head up with eyes closed; HF, head down with eyes closed. Mean \pm Standard Error.

kinematics. According to Quint and Toomey [25], these results indicate that the movements of a horse at a walking pace can facilitate pelvic movement in individuals with cerebral palsy. They theorized that the simulated riding employed in their study may have stretched subjects' tight pelvic and hip muscles, but it also may have facilitated more normalized movement of the pelvis while on the mechanical apparatus and enhanced the subject's feed-forward and feedback mechanisms of control over the pelvis [25]. However, in the present study, the SI showed no significant improvement in all test positions. In other words, the WDI scores of subjects with level 3 - 4 injuries and with Brown-Sequard syndrome increased significantly. Slow and rhythmic movement combined with a gentle stretching of stiff leg muscles appears to reduce abnormally high muscle tone and promote relation. Lechner et al. also reported that hippotherapy significantly reduced lower extremity spasticity in individuals with spinal cord injuries, at least for the short term [14,18]. The authors evaluated and compared spasticity using Ashworth scores and the VAS before and after hippotherapy sessions. When patients with spinal cord injuries walk and stand, spasticity increases their standing stability since spasticity is accompanied by muscle hypertonus, providing a "spastic brace" effect. Thus, spasticity has a particularly strong effect on the 
Table 3. The index of standing balance (F5-6, F7-8) before and after hippotherapy in patients with incomplete cervical spinal cord injury.

\begin{tabular}{|c|c|c|c|c|c|c|c|c|c|c|}
\hline & \multicolumn{10}{|c|}{ F5-6 (0.75 - 0.10) Hz } \\
\hline & \multicolumn{2}{|c|}{ Total } & \multicolumn{2}{|c|}{ C3-4 } & \multicolumn{2}{|c|}{ C3-4, CCS } & \multicolumn{2}{|c|}{ C3-4, BSS } & \multicolumn{2}{|c|}{ C5-6 } \\
\hline & \multicolumn{2}{|c|}{ Hippotherapy } & \multicolumn{2}{|c|}{ Hippotherapy } & \multicolumn{2}{|c|}{ Hippotherapy } & \multicolumn{2}{|c|}{ Hippotherapy } & \multicolumn{2}{|c|}{ Hippotherapy } \\
\hline & Before & After & Before & After & Before & After & Before & After & Before & After \\
\hline NO & $4.4 \pm 1.8$ & $3.8 \pm 1.3$ & $4.2 \pm 1.5$ & $3.8 \pm 1.4$ & $4.9 \pm 1.9$ & $4.1 \pm 1.7$ & $4.9 \pm 1.9$ & $4.1 \pm 1.7$ & $4.7 \pm 1.4$ & $3.7 \pm 1.2$ \\
\hline NC & $8.5 \pm 3.0$ & $7.9 \pm 2.6$ & $8.7 \pm 2.9$ & $8.3 \pm 2.4$ & $8.8 \pm 3.0$ & $10.5 \pm 2.5$ & $8.8 \pm 2.9$ & $10.5 \pm 2.5$ & $8.2 \pm 3.4$ & $7.2 \pm 2.9$ \\
\hline $\mathrm{PO}$ & $4.3 \pm 1.4$ & $3.8 \pm 1.2$ & $4.5 \pm 1.5$ & $3.9 \pm 1.36$ & $5.8 \pm 1.7$ & $3.6 \pm 0.7^{*}$ & $5.8 \pm 1.7$ & $3.6 \pm 0.7$ & $3.9 \pm 1.2$ & $3.7 \pm 1.1$ \\
\hline $\mathrm{PC}$ & $11.0 \pm 3.5$ & $11.2 \pm 3.7$ & $10.9 \pm 3.9$ & $11.8 \pm 3.8$ & $10.8 \pm 4.0$ & $11.7 \pm 2.9$ & $10.8 \pm 4.0$ & $11.7 \pm 2.9$ & $11.1 \pm 3.0$ & $10.2 \pm 3.5$ \\
\hline HR & $7.4 \pm 2.9$ & $7.2 \pm 3.0$ & $7.0 \pm 2.7$ & $7.8 \pm 3.2$ & $6.6 \pm 2.3$ & $7.8 \pm 2.4$ & $6.6 \pm 2.3$ & $7.8 \pm 2.4$ & $8.2 \pm 3.4$ & $6.2 \pm 2.5$ \\
\hline HL & $7.7 \pm 3.4$ & $6.9 \pm 3.0$ & $7.6 \pm 3.0$ & $7.2 \pm 3.0$ & $7.5 \pm 1.8$ & $8.7 \pm 2.5$ & $7.5 \pm 1.8$ & $8.7 \pm 2.5$ & $7.8 \pm 4.2$ & $6.4 \pm 3.1$ \\
\hline HB & $7.8 \pm 2.8$ & $7.1 \pm 2.4$ & $7.7 \pm 3.0$ & $7.4 \pm 2.3$ & $7.3 \pm 0.6$ & $7.9 \pm 1.6$ & $7.3 \pm 0.6$ & $7.9 \pm 1.6$ & $8.1 \pm 2.6$ & $6.4 \pm 2.5$ \\
\hline \multirow[t]{5}{*}{$\mathrm{HF}$} & $7.4 \pm 2.5$ & $6.9 \pm 3.2$ & $7.3 \pm 2.2$ & $7.3 \pm 3.2$ & $7.0 \pm 1.2$ & $8.9 \pm 1.8$ & $7.0 \pm 1.2$ & $8.9 \pm 1.8$ & $7.5 \pm 3.1$ & $6.3 \pm 3.3$ \\
\hline & \multicolumn{10}{|c|}{ F7-8 $(0.10-3.00) \mathrm{Hz}$} \\
\hline & \multicolumn{2}{|c|}{ Total } & \multicolumn{2}{|c|}{ C3-4 } & \multicolumn{2}{|c|}{ C3-4, CCS } & \multicolumn{2}{|c|}{ C3-4, BSS } & \multicolumn{2}{|c|}{ C5-6 } \\
\hline & \multicolumn{2}{|c|}{ Hippotherapy } & \multicolumn{2}{|c|}{ Hippotherapy } & \multicolumn{2}{|c|}{ Hippotherapy } & \multicolumn{2}{|c|}{ Hippotherapy } & \multicolumn{2}{|c|}{ Hippotherapy } \\
\hline & Before & After & Before & After & Before & After & Before & After & Before & After \\
\hline NO & $0.7 \pm 0.5$ & $0.7 \pm 0.6$ & $0.7 \pm 0.5$ & $0.8 \pm 0.6$ & $0.6 \pm 0.5$ & $0.6 \pm 0.6$ & $0.8 \pm 0.6$ & $0.9 \pm 0.6$ & $0.6 \pm 0.5$ & $0.6 \pm 0.5$ \\
\hline NC & $1.2 \pm 1.0$ & $1.2 \pm 1.1$ & $1.2 \pm 1.0$ & $1.3 \pm 1.7$ & $1.1 \pm 1.0$ & $1.3 \pm 1.2$ & $1.2 \pm 1.0$ & $1.3 \pm 1.2$ & $1.1 \pm 1.0$ & $1.0 \pm 0.9$ \\
\hline $\mathrm{PO}$ & $0.9 \pm 0.7$ & $0.8 \pm 0.6$ & $0.9 \pm 0.7$ & $0.8 \pm 0.7$ & $1.0 \pm 0.8$ & $0.9 \pm 0.9$ & $0.8 \pm 0.6$ & $0.8 \pm 0.6$ & $0.9 \pm 0.7$ & $0.8 \pm 0.6$ \\
\hline PC & $1.7 \pm 1.4$ & $1.7 \pm 1.6$ & $1.8 \pm 1.5$ & $1.9 \pm 1.8$ & $1.5 \pm 1.2$ & $1.8 \pm 1.4$ & $1.9 \pm 1.6$ & $2.0 \pm 2.0$ & $1.5 \pm 1.3$ & $1.3 \pm 1.1$ \\
\hline HR & $1.1 \pm 0.9$ & $1.1 \pm 0.9$ & $1.1 \pm 0.9$ & $1.2 \pm 1.0$ & $1.0 \pm 0.7$ & $1.0 \pm 0.8$ & $1.2 \pm 1.0$ & $1.3 \pm 1.1$ & $1.1 \pm 1.0$ & $0.9 \pm 0.9$ \\
\hline HL & $1.1 \pm 0.9$ & $1.0 \pm 0.9$ & $1.1 \pm 1.0$ & $1.2 \pm 1.0$ & $1.0 \pm 0.8$ & $0.9 \pm 0.8$ & $1.2 \pm 1.1$ & $1.2 \pm 1.1$ & $1.1 \pm 0.9$ & $0.8 \pm 0.7$ \\
\hline HB & $1.3 \pm 1.1$ & $1.1 \pm 1.1$ & $1.4 \pm 1.1$ & $1.3 \pm 1.1$ & $1.2 \pm 1.0$ & $1.2 \pm 1.1$ & $1.5 \pm 1.2$ & $1.3 \pm 1.2$ & $1.2 \pm 1.0$ & $1.0 \pm 0.9$ \\
\hline $\mathrm{HF}$ & $1.2 \pm 1.0$ & $1.1 \pm 1.1$ & $1.3 \pm 1.2$ & $1.2 \pm 1.2$ & $1.4 \pm 1.3$ & $1.2 \pm 1.1$ & $1.3 \pm 1.2$ & $1.3 \pm 1.3$ & $0.9 \pm 0.8$ & $0.8 \pm 0.7$ \\
\hline
\end{tabular}

Abbreviations: CCS, central cord syndrome; BSS, Brown-Sequard syndrome; NO, head straight with eyes open; NC, head straight with eyes closed; PO, standing on elastic pads with eyes open; PC, standing on elastic pads with eyes closed; HR, head right with eyes closed; HL, head left with eyes closed; HB, head up with eyes closed; HF, head down with eyes closed. Mean \pm Standard Error; ${ }^{*} \mathrm{p}<0.05$.

standing ability of patients with spinal cord injuries, as they have weak extremities [26]. According to these results it may be hard to maintain the positive effects of hippotherapy, including patients' reduced tightness, relaxation, and spasticity in their lumbar-hip-pelvic muscles in a standing position. Unfortunately, we did not analyze lumbar-hip-pelvic spasticity and muscular activity in this study. Therefore, future research should be done to provide further clarification. According to Heine [27], greater pelvic displacement increases somatosensory input though the joints and muscles during hippotherapy. Also, the movement through space involved in hippotherapy also provides a constantly changing vis- ual flow and increases the demands placed on an individual's vestibular system $[27,28]$. In the present study, subjects neurologic of level of 3 - 4 with central cord syndrome showed significant decreases at a frequency range of F5-6 in test position PO as well as on their WDI scores in test position PC. In the test positions PO and PC, the somatosensory input was typically blocked, indicating that the activation of the vestibular system may compensate for somatosensory deficits. Thus, hippotherapy seems to activate the vestibular system to help patients maintain balance in a standing position. In previous studies, balance and postural control were achieved as a result of integrating various multisensory processes. In 
Table 4. The index of standing balance (WDI, SI) before and after hippotherapy in patients with incomplete cervical spinal cord injury.

\begin{tabular}{|c|c|c|c|c|c|c|c|c|c|c|}
\hline & \multicolumn{10}{|c|}{ Weight Distribution Index (WDI) } \\
\hline & \multicolumn{2}{|c|}{ Total } & \multicolumn{2}{|c|}{ C3-4 } & \multicolumn{2}{|c|}{ C3-4, CCS } & \multicolumn{2}{|c|}{ C3-4, BSS } & \multicolumn{2}{|c|}{ C5-6 } \\
\hline & \multicolumn{2}{|c|}{ Hippotherapy } & \multicolumn{2}{|c|}{ Hippotherapy } & \multicolumn{2}{|c|}{ Hippotherapy } & \multicolumn{2}{|c|}{ Hippotherapy } & \multicolumn{2}{|c|}{ Hippotherapy } \\
\hline & Before & After & Before & After & Before & After & Before & After & Before & After \\
\hline NO & $8.2 \pm 3.5$ & $7.8 \pm 2.3$ & $7.3 \pm 1.3$ & $7.8 \pm 1.4$ & $7.5 \pm 1.2$ & $7.9 \pm 2.2$ & $7.2 \pm 1.4$ & $7.8 \pm 1.3$ & $9.7 \pm 5.7$ & $7.8 \pm 3.7$ \\
\hline NC & $8.6 \pm 3.2$ & $8.4 \pm 2.6$ & $9.0 \pm 3.3$ & $8.4 \pm 2.1$ & $7.2 \pm 0.8$ & $7.8 \pm 1.4$ & $9.7 \pm 3.8$ & $8.7 \pm 2.4$ & $7.9 \pm 3.3$ & $8.2 \pm 3.8$ \\
\hline $\mathrm{PO}$ & $6.7 \pm 2.6$ & $8.4 \pm 3.7$ & $6.5 \pm 1.7$ & $8.0 \pm 3.4$ & $6.2 \pm 1.5$ & $8.3 \pm 1.7$ & $6.6 \pm 1.9$ & $7.9 \pm 4.1$ & $7.2 \pm 4.1$ & $9.0 \pm 4.6$ \\
\hline PC & $7.1 \pm 2.4$ & $7.8 \pm 3.4$ & $6.6 \pm 1.8$ & $7.3 \pm 2.8$ & $8.1 \pm 1.3$ & $5.8 \pm 0.3^{*}$ & $6.0 \pm 1.7$ & $7.9 \pm 3.2$ & $8.0 \pm 3.3$ & $8.6 \pm 4.6$ \\
\hline HR & $7.6 \pm 3.0$ & $8.8 \pm 3.5$ & $7.1 \pm 2.5$ & $9.1 \pm 3.0$ & $8.6 \pm 0.6$ & $7.8 \pm 0.1$ & $6.5 \pm 2.8$ & $9.6 \pm 3.5$ & $8.6 \pm 4.1$ & $8.4 \pm 4.7$ \\
\hline HL & $8.9 \pm 4.1$ & $9.8 \pm 3.7$ & $9.4 \pm 4.5$ & $10.6 \pm 3.3$ & $9.6 \pm 2.4$ & $8.3 \pm 0.0$ & $9.3 \pm 5.4$ & $11.5 \pm 3.5$ & $8.0 \pm 3.8$ & $8.3 \pm 4.5$ \\
\hline HB & $7.8 \pm 2.9$ & $9.4 \pm 4.2$ & $7.9 \pm 2.6$ & $10.4 \pm 4.1$ & $9.6 \pm 0.8$ & $8.4 \pm 0.4$ & $7.3 \pm 2.8$ & $11.2 \pm 4.8$ & $7.6 \pm 4.0$ & $7.6 \pm 4.2$ \\
\hline \multirow[t]{5}{*}{$\mathrm{HF}$} & $7.0 \pm 3.5$ & $8.9 \pm 2.5$ & $6.7 \pm 3.7$ & $9.1 \pm 1.5$ & $8.1 \pm 0.5$ & $8.7 \pm 0.0$ & $6.1 \pm 4.3$ & $9.3 \pm 1.9$ & $7.6 \pm 3.7$ & $8.4 \pm 4.0$ \\
\hline & \multicolumn{10}{|c|}{ Stability Index (SI) } \\
\hline & \multicolumn{2}{|c|}{ Total } & \multicolumn{2}{|c|}{ C3-4 } & \multicolumn{2}{|c|}{ C3-4, CCS } & \multicolumn{2}{|c|}{ C3-4, BSS } & \multicolumn{2}{|c|}{ C5-6 } \\
\hline & \multicolumn{2}{|c|}{ Hippotherapy } & \multicolumn{2}{|c|}{ Hippotherapy } & \multicolumn{2}{|c|}{ Hippotherapy } & \multicolumn{2}{|c|}{ Hippotherapy } & \multicolumn{2}{|c|}{ Hippotherapy } \\
\hline & Before & After & Before & After & Before & After & Before & After & Before & After \\
\hline NO & $22.2 \pm 5.3$ & $20.2 \pm 5.2$ & $44.5 \pm 5.9$ & $45.1 \pm 12.1$ & $24.3 \pm 1.6$ & $23.7 \pm 6.6$ & $21.3 \pm 5.2$ & $21.2 \pm 4.5$ & $22.2 \pm 7.3$ & $18.6 \pm 5.9$ \\
\hline NC & $43.5 \pm 8.9$ & $42.9 \pm 12.6$ & $22.2 \pm 4.5$ & $21.9 \pm 4.7$ & $39.7 \pm 6.3$ & $53.2 \pm 9.7$ & $46.4 \pm 5.0$ & $41.9 \pm 12.4$ & $41.8 \pm 13.9$ & $39.1 \pm 14.4$ \\
\hline $\mathrm{PO}$ & $27.0 \pm 5.5$ & $24.6 \pm 5.1$ & $26.9 \pm 6.6$ & $25.3 \pm 5.4$ & $31.6 \pm 1.6$ & $26.0 \pm 4.0$ & $25.0 \pm 7.0$ & $25.0 \pm 6.2$ & $27.3 \pm 3.9$ & $23.4 \pm 5.3$ \\
\hline PC & $58.4 \pm 10.1$ & $57.6 \pm 17.9$ & $59.2 \pm 10.5$ & $62.1 \pm 20.7$ & $53.2 \pm 11.7$ & $57.0 \pm 0.9$ & $61.6 \pm 10.2$ & $64.2 \pm 25.0$ & $57.0 \pm 10.8$ & $48.6 \pm 8.6$ \\
\hline HR & $39.7 \pm 10.5$ & $38.0 \pm 11.3$ & $39.5 \pm 9.7$ & $39.6 \pm 11.9$ & $31.9 \pm 1.2$ & $37.1 \pm 3.0$ & $42.6 \pm 1.0$ & $40.6 \pm 14.3$ & $39.9 \pm 13.5$ & $35.1 \pm 11.2$ \\
\hline HL & $39.8 \pm 13.2$ & $35.4 \pm 14.3$ & $40.0 \pm 16.1$ & $38.0 \pm 16.2$ & $35.5 \pm 3.3$ & $38.8 \pm 2.5$ & $41.7 \pm 19.2$ & $37.3 \pm 19.7$ & $39.7 \pm 8.1$ & $30.7 \pm 10.7$ \\
\hline HB & $42.3 \pm 11.2$ & $40.3 \pm 11.3$ & $42.6 \pm 13.2$ & $42.8 \pm 12.1$ & $38.7 \pm 0.5$ & $40.0 \pm 4.5$ & $44.1 \pm 15.9$ & $44.0 \pm 14.5$ & $41.8 \pm 8.3$ & $36.0 \pm 9.6$ \\
\hline $\mathrm{HF}$ & $41.2 \pm 9.9$ & $37.8 \pm 13.9$ & $42.8 \pm 11.4$ & $41.7 \pm 15.5$ & $47.0 \pm 4.5$ & $45.0 \pm 2.2$ & $41.1 \pm 13.2$ & $40.4 \pm 18.7$ & $38.3 \pm 7.1$ & $31.1 \pm 8.3$ \\
\hline
\end{tabular}

Abbreviations: CCS, central cord syndrome; BSS, Brown-Sequard syndrome; NO, head straight with eyes open; NC, head straight with eyes closed; PO, standing on elastic pads with eyes open; PC, standing on elastic pads with eyes closed; HR, head right with eyes closed; HL, head left with eyes closed; HB, head up with eyes closed; HF, head down with eyes closed. Mean \pm Standard Error; ${ }^{*} \mathrm{p}<0.05$.

the present study, rather than activating the somatosensory system, hippotherapy treatment primarily activated the vestibular system for standing balance. Also, based on the present findings, movement of the lumbar-hippelvic complex immediately after hippotherapy appears to be hard to maintain in a standing position. The present study trained hippotherapy. Improved particularly standing balance, but the present study resulted in cannot improve standing balance with incomplete cervical spinal cord injury. In the present investigation, our goals were realized in that subjects improved their standing balance, decreased their risk of falling, and increased their activities of daily living. However, the small number of par- ticipants, the short intervention period, and the lack of concern about spasticity in the present study make it difficult to state with confidence that hippotherapy would improve the standing balance of all patients with incomeplete cervical spinal cord injuries. Therefore, further studies that include longer intervention periods are necessary.

\section{REFERENCES}

[1] R. Bailey, V. Kaskutas, I. Fox, C. M. Baum and S. E. Mackinnon, "Effect of Upper Extremity Nerve Damage on Activity Participation, Pain, Depression, and Quality of Life,” Journal of Hand Surgery, Vol. 34, No. 9, 2009, 
14 The Effects of Hippotherapy on Standing Balance in Patients with Incomplete Cervical Spinal Cord Injuries: A Pilot Study

pp. 1682-1688. doi:10.1016/j.jhsa.2009.07.002

[2] C. Fekete and A. Rauch, "Correlates and Determinants of Physical Activity in Persons with Spinal Cord Injury: A Review Using the International Classification of Functioning, Disability and Health as Reference Framework," Disability and Health Journal, Vol. 5, No. 3, 2012, pp. 140-150. doi:10.1016/j.dhjo.2012.04.003

[3] A. D. Nichol, A. M. Higgins, B. J. Gabbe, L. J. Murray, D. J. Cooper and P. A. Cameron, "Measuring Functional and Quality of Life Outcomes Following Major Head Injury: Common Scales and Checklists,” Injury, Vol. 42, No. 3, 2011, pp. 281-287. doi:10.1016/j.injury.2010.11.047

[4] P. Krawetz and P. Nanace, "Gait Analysis Spinal Cord Injured Subjects: Effects of Injury Level and Spasticity,” Archives of Physical Medicine and Rehabilitation, Vol. 77, No. 7, 1996, pp. 635-638. doi:10.1016/S0003-9993(96)90000-3

[5] R. Kizony, L. Raz, N. Katz, H. Weingarden and L. T. Weiss, "Video-Capture Virtual Reality System for Patients with Paraplegic Spinal Cord Injury,” Journal of Rehabilitation Research \& Development. Vol. 42, No. 5, 2005, pp. 595-608. doi:10.1682/JRRD.2005.01.0023

[6] N. Benjuya, I. Melzer and J. Kaplanski, “Aging-Induced Shifts from a Reliance on Sensory Input to Muscle Cocontraction during Balanced Standing," The Journals of Gerontology, Vol. 59, No. 2, 2004, pp. 166-171.

[7] S. Wydenkeller, M. Liechti, R. Muller and A. Curt, "Impaired Scaling of Responses to Vestibular Stimulation in Incomplete SCI,” Experimental Brain Research, Vol. 175, No. 1, 2006, pp. 191-195. doi:10.1007/s00221-006-0688-z

[8] S. D. Anderson, D. G. Anderson and A. R. Vaccaro, "Skeletal Fracture Demographics in Spinal Cord-Injured Patients," Archives of Orthopaedic and Trauma Surgery, Vol. 124, No. 3, 2004, pp. 193-196. doi:10.1007/s00402-003-0605-X

[9] D. S. Marigold, J. J. Eng, A. S. Dawson, J. T. Inglis, J. E. Harris and S. Gylfadóttir, "Exercise Leads to Faster Postural Reflexes, Improved Balance and Mobility, and Fewer Falls in Older Persons with Chronic Stroke,” Journal of the American Geriatrics Society, Vol. 53, No. 3, 2005, pp. 416-423. doi:10.1111/j.1532-5415.2005.53158.x

[10] F. P. Carpes, F. B. Reinehr and C B. Mota, "Effects of a Program for Trunk Strength and Stability on Pain, Low Back and Pelvis Kinematics, and Body Balance: A Pilot Study," Journal of Bodywork and Movement Therapies, Vol. 12, No. 1, 2008, pp. 22-30. doi:10.1016/j.jbmt.2007.05.001

[11] S. F. Nadler, G. A. Malanga, L. A. Bartoli, J. H. Feinberg, M. Prybicien and M. Deprince, "Hip Muscle Imbalance and Low-Back Pain in Athletes: Influence of Core Strengthening," Medicine \& Science in Sports \& Exercise, Vol. 34, No. 1, 2002, pp. 9-16. doi:10.1097/00005768-200201000-00003

[12] J. S. Petrofsky, E. G. Johnson, A. Hanson, M. Cuneo, R. Dial, R. Somers, G. De La Torre, A. Martinez, M. McKenzie and B. Forrester, "Abdominal and Lower Back
Training for People with Disabilities Using a 6 Second abs Machine: Effect on Core Muscle Stability,” The Journal of Applied Research, Vol. 5, No. 2, 2005, pp. 345359.

[13] P. Herrero, A. Asensio, E. Garcia, A. Marco, B. Olivan and A. Ibarz, "Study of the Therapeutic Effects of an Advanced Hippotherapy Simulator in Children with Cerebral Palsy: A Randomized Controlled Trial,” BMC Musculoskeletal Disorders, Vol. 11, 2010, p. 71. doi:10.1186/1471-2474-11-71

[14] H. E. Lechner, T. H. Kakebeeke, D. Hegemann and M. Baumberger, "The Effect Hippotherapy on Spasticity and on Mental Well-Being of Persons with Spinal Cord Injury," Archives of Physical Medicine and Rehabilitation, Vol. 88, No. 10, 2007, pp. 1241-1248. doi:10.1016/j.apmr.2007.07.015

[15] D. Silkwood-Sherer and H. Warmbier, "Efeects of Hippotherapy on Postural Stability, in Persons with Multiple Sclerosis: A Pilot Study,” Journal of Neurologic Physical Therapy, Vol. 31, No. 2, 2007, pp. 77-84.

[16] J. L. Encheff, C. Armstrong, M. Masterson, C. Fox and P. Gribble, "Hippotherapy Effects on Trunk, Pelvic, and Hip Motion during Ambulation in Children with Neurological Impairments,” Pediatric Physical Therapy, Vol. 24, No. 3, 2012, pp. 242-250. doi:10.1097/PEP.0b013e31825c1dc3

[17] D. B. Bertoti, "Effect of Therapeutic Horseback Riding on Posture in Children with Cerebral Palsy," Physical Therapy, Vol. 68, No. 10, 1998, pp. 1505-1512.

[18] H. E. Lechner, S. Feldhaus, L. Gudmundsen, D. Hegemann, D. Michel, G. A. Zach and H. Knecht, "The ShoetTerm Effect of Hippotherapy on Spasticity in Patients with Spinal Cord Injury,” Spinal Cord, Vol. 41, No. 9, 2003, pp. 502-505. doi:10.1038/sj.sc.3101492

[19] F. M. Maynard Jr., M. B. Bracken, G. Creasey, J. F. Ditunno Jr., W. H. Donovan, T. B. Ducker, S. L. Garber, R. J. Marino, S. L. Stover, C. H. Tator, R. L. Waters, J. E. Wilberger and W. Young, "International Standards for Neurological and Functional Classification of Spinal Cord Injury. American Spinal Injury Association,” Spinal Cord, Vol. 35, No. 5, 1997, pp. 266-274. doi:10.1038/sj.sc.3100432

[20] U. Oppenheim, A. Kohen-Raz, R. Kohen-Raz, M. Azarya and D. Alex, "Postural Characteristics of Diabetic Neuropathy," Diabetes Care, Vol. 22, No. 2, 1999, pp. 328332. doi:10.2337/diacare.22.2.328

[21] M. Friedrich, H. J. Grein, C. Wicher, J. Schuetze, A. Mueller, A. Lauenroth, K. Hottenrott and R. Schwesig, "Infuence of Pathologic and Simulated Visual Dysfunctions on the Postural System," Experimental Brain Research, Vol. 186, No. 2, 2008, pp. 305-314. doi:10.1007/s00221-007-1233-4

[22] S. Schwartz, O. Segal, Y. Barkana, R. Schwesig, I. Avni and Y. Morad, "The Effect of Cataract Surgery on Postural Control," Investigative Ophthalmology \& Visual Science, Vol. 46, No. 3, 2005, pp. 920-924. doi:10.1167/iovs.04-0543

[23] A. Hammer, Y. Nilsagard, A. Forsberg, H. Pepa, E. Skargren and B. Oberg, "Evaluation of Therapeutic Riding (Sweden)/Hippotherapy (United States). A Single-Subject 
Experimental Design Study Replicated in Eleven Patients with Multiple Sclerosis,” Physiotherapy Theory and Practice, Vol. 21, No. 1, 2005, pp. 51-77. doi:10.1080/09593980590911525

[24] C. Manella and D. Backus, "Gait Characteristics, Range of Motion, and Spasticity Changes in Response to Massage in a Person with Incomplete Spinal Cord Injury: Case Report," International Journal of Therapeutic Massage \& Bodywork. Vol. 4, No. 1, 2011, pp. 28-39.

[25] C. Quint and M. Toomey, "Powered Saddle and Pelvic Mobility: An Investigation into the Effects on Pelvic Mobility of Children with Cerebral Palsy of a Powered Saddle which Imitates the Movements of a Walking Horse," Physiotherapy, Vol. 84, No. 8, 1998, pp. 376-384. doi:10.1016/S0031-9406(05)61458-7
[26] T. Suzuki, S. Sonoda, E. Saitoh, K. Onogi, H. Fujino and T. Teranishi, "Prediction of Gait Outcome with the KneeAnkle-Foot Orthosis with Medial Hip Joint in Patients with Spinal Cord Injuries: A Study Using Recursive Partitioning Analysis,” Spinal Cord, Vol. 45, No. 1, 2007, pp. 57-63. doi:10.1038/sj.sc.3101955

[27] B. Heine, "Hippotherapy. A multisystem Approach to the Treatment of Neuromuscular Disorder," Australian Journal of Physiotherapy, Vol. 43, No. 2, 1997, pp. 145-149.

[28] S. Muñoz-Lasa, G. Ferriero, R. Valero, F. Gomez-Muñiz, A. Rabini and E. Varela, "Effect of Therapeutic Horseback Riding on Balance and Gait of People with Multiple Sclerosis,” Giornale Italiano di Medicina del Lavoro ed Ergonomia, Vol. 33, No. 4, 2011, pp. 462-467. 